\title{
Remote magnetic catheter navigation: more than just bells and whistles?
}

\author{
N. M. S. de Groot
}

Published online: 8 May 2013

(C) The Author(s) 2013. This article is published with open access at Springerlink.com

Catheter ablation is nowadays an established treatment modality for both atrial and ventricular tachyarrhythmias. Manual navigation of the ablation catheter can be challenging in patients with a complex cardiac anatomy due to, for example, (surgically corrected or palliated) congenital heart defects, resulting in a long procedure time and excessive X-ray exposure. In the past decades, sophisticated mapping and ablation techniques have been developed in order to improve the outcome of ablative therapy. These technologies include remote navigation systems such as the magnetic navigation system (Stereotaxis Inc.) and the non-magnetic robotic navigation system (Sensei Hansen Medical).

In this issue, Roudijk et al. report on the value of remote magnetic catheter navigation and ablation in children and young adult patients with and without congenital heart disease in their paper entitled: 'Catheter ablation in children and young adults: is there an additional benefit from remote magnetic navigation [1]? In this elegant paper, the outcome of ablative therapy of a variety of tachyarrhythmias was examined.

\section{Remote catheter navigation}

Numerous investigators have already examined the potential benefits of remote (non)-magnetic robotic navigation systems for ablation of both atrial and ventricular tachycardias [2]. The major advantage of the remote magnetic navigation system is its 'floppy' ablation catheter. Because of this floppiness, there is an enormous freedom of movement of the ablation catheter. The operator can easily reach any desirable site on the endocardium or epicardium due to the absence of a predefined curve. As the atraumatic catheter design is less harmful to the

\section{N. M. S. de Groot $(\triangle)$}

Department of Cardiology, Erasmus Medical Center,

Thorax Center BA579 Erasmus MC's Gravendijkwal 230,

3015CE Rotterdam, the Netherlands

e-mail: nmsdegroot@yahoo.com cardiac wall, this ablation technology can also be safely used by less experienced operators. However, lesion formation by this soft ablation catheter may be inadequate. The remote nonmagnetic robotic navigation system (Sensei Hansen Medical) does not have this limitation. This system consists of a standard ablation catheter integrated into a remote steerable double sheath, a robotic arm and a workstation equipped with a tactile feedback system. It has been shown that remote robotic navigation technology combined with contact force sensing does result in transmural ablation lesions.

Previous studies have demonstrated that general advantages of remote (non)-magnetic robotic navigation systems for ablation of a variety of tachyarrhythmias include reduction in total procedure time, radiofrequency time, number of radiofrequency applications, improved catheter stability and steerability, and reduction in fluoroscopy time not for only the doctor, but also for the patient [3]. Reduction in radiation exposure from fluoroscopy is particularly desirable in young patients, as there is a risk for radiation-induced cancer.

\section{Are there true benefits of remote magnetic catheter navigation?}

The importance of reduction in radiation exposure in paediatric and young adult patients is well recognised by Roudijk et al. [1]. They compared manual navigation and remote magnetic navigation in 62 young patients with and without congenital heart disease. Compared with conventional catheter navigation, a larger number of patients (median age 19.7) undergoing ablative therapy guided by remote magnetic catheter navigation had congenital heart disease (67\% versus $37 \%$ ) or prior cardiac surgery (59\% versus $20 \%$ ). Usage of a remote magnetic navigation system resulted in less fluoroscopy time despite a longer procedure time. Except for one puncture-related complication, there were no complications related to the mapping and/or ablation procedure. Based on 
these findings, the authors concluded that catheter ablation using remote magnetic navigation is safe and feasible, particularly in paediatric patients and young adults. Though the concept of this study is interesting and of paramount importance, the data provided by this study should be interpreted with caution.

As also stated by the authors, both groups contain only a small number of patients, with diversity in underlying cardiac anatomy and a variety of tachycardias; comparison of the two groups is therefore difficult.

In patients with congenital heart defects, the arrhythmogenic substrate is usually complex due to the presence of multiple areas of scar tissue or surgically created barriers. In these patients, extensive mapping of the heart chamber of interest is essential to comprehend the mechanism underlying the arrhythmia. This may result in prolonged radiation exposure. Unfortunately, the authors do not provide detailed information on the complexity of the arrhythmogenic substrate. For example, the number of mapping points necessary to identify target sites for ablation - as an indicator of the complexity of the arrhythmia would be interesting. If the number of mapping points required is the same for each group but less fluoroscopy time was used in the remote magnetic navigation system group it would further support the benefits of this technology. In addition, both groups contain a considerable number of patients with atrioventricular reentrant tachycardias or atrioventricular nodal reentrant tachycardias that do rarely require electroanatomical mapping prior to ablation.

The follow-up period in this study is relatively short (only 6 months) to evaluate long-term success. However, patients with surgically corrected or palliated congenital heart defects often have 'recurrences' of tachycardias which are usually new tachycardias due to progression of the cardiomyopathy instead of an unsuccessful ablation procedure [4].

As the ablation catheter used for remote magnetic navigation is soft and floppy, it is only logical to assume that catheter manipulation is safer than manual catheter navigation. Yet, as other studies reporting on the outcome of ablative therapy in paediatric patients and young adult patients using manually guided catheter navigation also report a low complication rate, it will be difficult to demonstrate that the outcome of ablative therapy guided by remote magnetic navigation is safer[5, 6].

Reduction in radiation exposure is the most important advantage of remote magnetic catheter navigation. In the study population of Roudijk et al. cardiac computed tomography was performed prior to ablation in four patients in order to facilitate the mapping procedure [1]. Hence, this imaging technique increases radiation exposure significantly and should be avoided whenever possible.

Nevertheless, the paper by Roudijk et al. is another important contribution to the evaluation of the benefits of remote magnetic-guided catheter ablation. Future trials as proposed by the authors will be of great value for further establishing the role of remote catheter navigation and ablation technologies in young patients with complex arrhythmias and determine whether remote magnetic catheter navigation is more than just bells and whistles.

Funding None.

Conflict of interests None declared.

Open Access This article is distributed under the terms of the Creative Commons Attribution License which permits any use, distribution, and reproduction in any medium, provided the original author(s) and the source are credited.

\section{References}

1. Roudijk RW, Gujic M, Suman-Horduna I, et al. Catheter ablation in children and young adults: is there an additional benefit from remote magnetic navigation? Neth Heart J. 2013. doi:10.1007/s12471-0130408-9 (Epub ahead of print).

2. Schmidt B, Chun KR, Tilz RR, et al. Remote navigation systems in electrophysiology. Europace. 2008;10:57-61.

3. Bradfield J, Tung R, Mandapati R, et al. Catheter ablation utilizing remote magnetic navigation: a review of applications and outcomes. Pacing Clin Electrophysiol. 2012;35:1021-34.

4. de Groot NM, Atary JZ, Blom NA, et al. Long-term outcome after ablative therapy of postoperative atrial tachyarrhythmia in patients with congenital heart disease and characteristics of atrial tachyarrhythmia recurrences. Circ Arrhythm Electrophysiol. 2010;3:14854.

5. Kolditz DP, Blom NA, Bökenkamp R, et al. Radiofrequency catheter ablation for treating children with cardiac arrhythmias: favourable results after a mean of 4 years. Ned Tijdschr Geneeskd. 2005;149:1339-46.

6. Tanel RE, Walsh EP, Triedman JK. Five-year experience with radiofrequency catheter ablation: implications for management of arrhythmias in pediatric and young adult patients. J Pediatr. 1997; 131:878-87. 